\title{
Treatment of a Breast Cancer with Pregnancy Preserving her Breast and Baby
}

\author{
PS AKHTAR $^{\mathrm{a}}$, MM RAHMAN $^{\mathrm{b}}, \mathrm{K}^{\text {NAHAR }}{ }^{\mathrm{c}}$ \\ MA ISLAM ${ }^{\mathrm{d}}$, SN DAY
}

(J Bangladesh Coll Phys Surg 2006; 24: 38-41)

\section{Introduction:}

Breast cancer is reported to occur in from 1 in 3000 to 1 in 10,000 pregnancies worldwide ${ }^{1}$. It may present many difficult medical and psychosocial problems and historically has placed the welfare of the mother in conflict with that of the fetus.

To detect breast cancer, pregnant and lactating women should practice self-examination and undergo a breast examination as part of the routine prenatal examination by a doctor. If an abnormality is found, diagnostic approaches such as ultrasound and mammography may be used. With proper shielding, mammography poses little risk of radiation exposure to the fetus ${ }^{2}$. Diagnosis may be safely accomplished with a fine-needle aspiration or excisional biopsy under local anesthesia ${ }^{3}$

Procedures used for staging of breast cancer should be modified to avoid radiation exposure to the fetus in pregnant women.

Once the diagnosis is established, pregnant patients should be treated in a manner similar to nonpregnant patients because there is no evidence that carcinoma of the breast in pregnant women is biologically different than carcinoma of the breast in other premenopausal women ${ }^{4}$.

Operation may be performed safely when general anesthesia is administered and postoperative adjuvant

a. Prof. Parveen Shahida Akhtar, Professor of Medical Oncology, National Institute of Cancer Research \& Hospital, Dhaka.

b. Dr. Md Mujibur Rahman, Associate Professor of Surgery, Mymensingh Medical College.

c. Dr. Kumrun Nahar, Associate Professor of Gynecology and Obstetrics, Mymensingh Medical College.

d. Dr. Md. Aminul Islam, Assistant Professor of Radiotherapy, Mymensingh Medical College.

e. Dr. Sampad Narayan Day, Radiotherapist, Mymensingh Medical College Hospital.

Address of correspondence: Prof. Parveen Shahida Akhtar, Professor of Medical Oncology, National Institute of Cancer Research \& Hospital, Mohakhali, Dhaka, Tel. 8111880 (Res), 0171622788 (m), e-mail: shanti@bangla.net,psakhtar@dhaka.net therapy should be administered, when necessary, with the exception of radiotherapy, which is contraindicated throughout pregnancy; and chemotherapy, which is contraindicated during the first trimester ${ }^{5}$. The involvement of multiple subspecialties in the management of these patients is highly recommended ${ }^{6}$.

Termination of pregnancy has not been shown to have any beneficial effect on breast cancer outcome and is not usually considered as a therapeutic option ${ }^{1}$. Termination of pregnancy, however, may be considered, based on the age of the fetus, and if maternal treatment options, such as chemotherapy and radiation therapy, are significantly limited by the continuation of the pregnancy.

Having to start chemotherapy during pregnancy remains a rare event. The decision to proceed with this treatment depends on the drugs used, the time of exposure for the fetus and the gestational age at the time of exposure. The mutagenic potential of the chosen drug has to be known. The risk for the child can then be established without compromising the mother's chances of survival or well-being. The first trimester of pregnancy, which corresponds to organogenesis is the most critical period for the fetus. The greatest risk of malformation occurs with antimetabolite drugs ${ }^{7}$. Neoadjuvant or adjuvant chemotherapy can be given with minimal risks to the fetus during the second or third trimester ${ }^{6}$.

The main goal of this article is to offer to the pregnant woman diagnosed with breast cancer the optimal therapeutic modalities, while protecting the unborn fetus of immediate and late deleterious effects of radiation and chemotherapy.

\section{Case history:}

A 29-year lady belonged to a well to do family of Mymensingh town attended Mymensigh Medical College Hospital on $4^{\text {th }}$ March 2000 with the complaints of a lump in her left breast and 
amenorrhoea for three months. Her last menstrual period was $25^{\text {th }}$ December 1999 . Her expected date of delivery was $2^{\text {nd }}$ October 2000 . The patient was educated (HSC), multipara (two children) and with excellent performance status (100\% Kernofsky). She was non diabetic and non hypertensive.

On local examination, there was a lump in the upper and outer quadrant of left breast, size about $2 \times 2 \mathrm{~cm}^{2}$, irregular shape, mobile and free from overlying skin and underlying chest wall.

No abnormality was detected in the other breast and the both axilla. On gynecological examination, uterus was 14 weeks size. For diagnostic work up and staging the following investigations routine blood with platelet count, blood chemistries such as fasting blood sugar, urea, creatinine, bilirubin, SGPT, alkaline phosphatase, routine urine examination, ultrasonograghy (USG) of both breasts, whole abdomen, X-ray chest PA view with proper shielding and fine needle aspiration cytology from the left breast lump were done.

USG of left breast showed a solid irregular lump size of $2 \times 2 \mathrm{~cm}$ in upper and outer quadrant and the fetus was 14 weeks size in the womb. FNAC revealed infiltrating duct cell carcinoma. All other investigations revealed the values within normal limit. The diagnosis was left breast cancer T1N0M0 clinically with 14 weeks pregnancy.

During the pregnancy she was treated by lumpectomy with axillary clearance on $18^{\text {th }}$ April, 2000. Histopathology of the breast lump showed poorly differentiated infiltrating duct cell carcinoma and three axillary lymph nodes showed the involvement of duct cell carcinoma. Her estrogen and progesterone receptor status could not be done. After complete healing of the surgical wound she was treated by combination chemotherapy with cyclophosphamide $900 \mathrm{mg}$ IV and doxorubicine $75 \mathrm{mg}$ IV three weekly for six cycles. Before each cycle of chemotherapy antenatal check up was done clinically, sonologically and other investigations such as routine blood, urine and blood chemistries. The patient was found fit for chemotherapy and the fetus was found normal height for gestational age, fetal movement and heart sound were within normal limit. On $25^{\text {th }}$ September 2000 she delivered a healthy male baby of $2.7 \mathrm{~kg}$ per vaginally. At birth the baby was found normal physically preserving all milestones. The mother was advised not to breast feed her baby because of exposure of chemotherapy.

Four weeks after delivery, the patient was treated by radiotherapy by cobalt 60 teletherapy machine with the dose 50Gy in 5 weeks in 25 fractions, 5 fractions per weeks $\left(30^{\text {th }}\right.$ October, 2000 to $10^{\text {th }}$ November 2000). Radiotherapy treatment was given in four portals; -two tangential fields, left medial tangential and left lateral tangential portals $(15 \mathrm{~cm} \times 9 \mathrm{~cm})$ covering the left breast (whole), third internal mammary portals $(8 \mathrm{~cm} \times 4 \mathrm{~cm})$ to irradiate the Internal mammary node and the fourth portal to the supraclavicular nodes $(9 \mathrm{~cm} \times 10 \mathrm{~cm})$. Before starting radiotherapy each portal was simulated by Simulator. She was advised for boost radiation further 12Gy in 6 fractions either by electron beam or interstitial implant (brachytherapy), but she refused.

The patient attended for follow up examination regularly, one follow up per two months for the first year and then four follow up per year. The baby was on close observation and monthly check up to three months and then mother and baby both attended at the same setting for follow up care. At the end of 40 months of diagnosis of breast cancer the patient was found disease free and the baby at his 33 months of age was with normal physical and mental growth (developmental milestone).

\section{Discussion:}

The study case was 29 year young lady. Fortunately she was diagnosed at early stage but at that time she was on $14^{\text {th }}$ week of pregnancy. During the pregnancy she was treated by lumpectomy with axillary clearance. Histopathology of the breast lump revealed infiltrating duct cell carcinoma poorly differentiated and there was involvement of axillary lymph nodes histopathologically. Breast cancer in women under 30 years of age carries a poor prognosis, for reasons that have not been identified ${ }^{7}$. Across stages, patients with pregnancy associated breast cancer have survival not significantly different from those patients with nonpregnancy-associated breast cancer ${ }^{8}$ but during pregnancy and lactation the prognosis is worse because of delay in diagnosis, average reported delay 5 to 15 months from the onset of symptoms ${ }^{3}$. 
Although breast conservation therapy has evolved as the major treatment in breast cancer, it has been thought that pregnancy was a contraindication for this type of breast cancer therapy due to risks imposed on the fetus by chemotherapy and radiation. When the cancer is detected in the second or third trimester, chemotherapy is initiated after a lumpectomy, radiation can be withheld until after the birth of the baby. In the present study, breast cancer was diagnosed at the second trimester of pregnancy, breast conserving surgery was done and chemotherapy was carried out during pregnancy and radiotherapy after four weeks the delivery of the baby. A study in Agios Andreas Hospital, Greece had shown a 32-year lady diagnosed as breast cancer with $19^{\text {th }}$ week pregnancy treated by quadrenectomy and axillary clearence and four cycles of chemotherapy with cyclophosphamide, pharmarubicine and 5 flourouracil combination during pregnancy $\left(2^{\text {nd }}\right.$ and $3^{\text {rd }}$ trimester) and the patient gave birth in the $35(+6)$ week to a healthy baby with no apparent malformations; during puerperium she received two more cycles of chemotherapy and then radiotherapy with tangential fields ${ }^{9}$

Postoperative chemotherapy is the standard treatment for node-positive pre menoposal women with breast cancer. As the study case was 29 years node positive and poorly differentiated duct cell carcinoma and chemotherapy during pregnancy is not absolute contraindication, so she was treated by adjuvant chemotherapy with cyclophophamide and adriamycine three weekly for 6 cycles during pregnancy at second and third trimester. A healthy male baby was born 15 days before the expected date of delivery. The patient was advised not to breast feed her baby. Up to 33 months of age the baby was found normal physically preserving all the developmental milestones. And the mother at $40^{\text {th }}$ month of her diagnosis of breast cancer was also found disease free.

Giacalone PL, Laffargue F, Benos P showed in their study "chemotherapy for breast cancer during pregnancy-a French national survey" $95 \%$ of the pregnancies resulted in live births with low related morbidity in the newborns and at a mean follow up of 42.3 months, all live infants were reported to have reached normal developmental milestones ${ }^{10}$. The best data for management of breast cancer during pregnancy come from the experience at the MD Anderson Cancer Center in Houston, Texas, in which 24 women were treated over an 8-year span using a standardized protocol ${ }^{11}$. After surgery (ie, modified radical mastectomy), patients received adjuvant chemotherapy with 5-fluorouracil, doxorubicin, and cyclophosphamide (FAC) until the time of delivery. There were no unexpected antepartum complications; $12 \%$ had preterm labor and $4 \%$ had pre-eclampsia. Postpartum lactation was impaired in all patients and patients were advised not to breast feed on account of chemotherapy exposure. None of the 24 children had congenital abnormalities; 23 of the 24 had birth weights above the 10th percentile. No unusual neonatal complications were noted, although 1 baby had transient leucopenia. So MD Anderson Cancer center recommendation is that treatment with chemotherapy during the first trimester is contraindicated, as studies show high rates of fetal side effects and treatment with tamoxifen or therapeutic radiation therapy is also contraindicated during pregnancy.

The study case was diagnosed as an early breast cancer at $2^{\text {nd }}$ trimester of pregnancy, breast conserving surgery followed by chemotherapy could be possible. A healthy baby was born at 34 weeks. Radiotherapy treatment after the delivery of the baby was executed. There was no adverse effect on baby in physical and developmental milestone up to 33 months of age and no local recurrence and metastasis of the mother were detected up to 40 months of the diagnosis of breast cancer.

\section{Conclusion:}

As the carcinoma of breast in pregnant women is not biologically different from the carcinoma of breast in other pre-menopausal women ${ }^{4}$, if the early breast cancer diagnosed at the $2^{\text {nd }}$ and $3^{\text {rd }}$ trimester of pregnancy is appropriately staged and adequately treated; preservation of breast and baby can be possible without compromising maternal survival.

\section{References:}

1. Petrek J, Seltzer V. Breast cancer in pregnant and postpartum women, J Obstet Gynaecol Can. 2003 Nov; 25(11): 944-50. 
2. Barnavon Y, Wallach MK. Management of the pregnant patient with carcinoma of the breast, Surgery,Gynecology and Obstetrics, 1990; 171(4): 347-352.

3. Novotny DB, Maygarden SJ, shermer RW, et al; Fine needle aspiration of benign and malignant masses associated with pregnancy, Acta Cytologica, 1991; 35(6): 676-686.

4. Barnavon Y, Wallack MK, Management of the pregnant patient with carcinoma of the breast, Surg Gynecol Obstet; 1990,171(4): 347-352.

5. Puckridge PJ, Saunders CM, Ives AD, Semmens JB. Breast cancer and pregnancy: a diagnostic and management dilemma. ANZ J Surg. 2003 Jul; 73(7): 500-3.

6. Keleher AJ, Theriault RL, Gwyn KM, Hunt KK, Stelling CB, Singletary SE, Ames FC, Buchholz TA, Sahin AA, Kuerer HM. Multidisciplinary management of breast cancer concurrent with pregnancy. J Am Coll Surg. 2002 Jan; 194(1): 54-64.7.
7. Guinee VF, Olsson H, Moller T, Hess KR, Taylor SH, Fahey T, Gladikov JV, van den Blink JW, Bonichon F, Dische S, et al. Effect of pregnancy on prognosis for young women with breast cancer. Lancet; 1994; 343(8913): 1587-1589.

8. Gwyn K, Theriault R: Breast cancer during pregnancy, Oncology, 2001; 15(1): 39-46.

9. Ginopoulos PV, Michail GD, Kourounis GS. Pregnancy associated breast cancer: a case report. Eur J Gynaecol Oncol. 2004; 25(2): 261-3.

10. Giacolone PL, Laffargue F, Benos P. Chemotherapy for breast carcinoma during pregnancy: a French national survey. Cancer. 1999; 86: 2266-2272.

11. Berry DL, Theriault RL, Holmes FA, et al. Management of breast cancer during pregnancy using a standardized protocol. J Clin Oncol. 1999; 17: 855-861. 